\title{
Stray Light Suppression in the Goddard IRAM 2-Millimeter Observer (GISMO)
}

\author{
E.H. Sharp, ${ }^{1,3}$ D.J. Benford, ${ }^{3}$ D.J. Fixsen, ${ }^{4,3}$ S.H. Moseley, ${ }^{3}$ J.G. Staguhn, ${ }^{2,3}$ E.J. Wollack ${ }^{3}$
}

1. Global Science \& Technology, Inc., 7855 Walker Drive, Suite 200, Greenbelt, MD 20770, USA

2. The Henry A Rowland Department of Physics and Astronomy, Johns Hopkins University, 3400 N. Charles Street, Baltimore, MD 21218, USA

3. Observational Cosmology Lab, Code 665, NASA Goddard Space Flight Center, Greenbelt, MD 20771, USA

4. CREEST, University of Maryland-College Park, College Park, MD 20742, USA

\begin{abstract}
The Goddard-IRAM Superconducting 2 Millimeter Observer (GISMO) is an 8x16 Transition Edge Sensor (TES) array of bolometers built as a pathfinder for TES detector development efforts at NASA Goddard Space Flight Center. GISMO has been used annually at the Institut de Radioastronomie Millimétrique (IRAM) 30 meter telescope since 2007 under engineering time and was opened in the spring of 2012 to the general astronomical community. The spring deployment provided an opportunity to modify elements of the room temperature optics before moving the instrument to its new permanent position in the telescope receiver cabin. This allowed for the possibility to extend the cryostat, introduce improved cold baffling and thus further optimize the stray light performance for final astronomical use of the instrument, which has been completed and validated. We will demonstrate and discuss several of the methods used to quantify and limit the influence of stray light in the GISMO camera.
\end{abstract}

Keywords: GISMO, Stray light, Bolometer, Transition Edge Sensor (TES)

\section{Introduction}

GISMO is optimized for the detection of extremely high red shift galaxies ${ }^{1}$ at a wavelength of $2 \mathrm{~mm}$. The camera ${ }^{2}$ is based upon a close packed 128-pixel TES sensor operating at $450 \mathrm{mK}$ in a Backshort Under Grid (BUG) architecture ${ }^{3}$. GISMO contains a $4.2 \mathrm{~K}$ anti-reflection coated silicon $\mathrm{len}^{4}$ as well as an appropriate set of cold band defining edge and thermal blocking filters ${ }^{5,6}$. The camera has a cryogenic filter mechanism that allows neutral density filters to be moved in and out of the beam depending on the observing conditions. This instrument's particular design feature was proven useful in understanding the observed detector excess power loading noted during the initial telescope-commissioning phase. GISMO employs a specially developed $2 \mathrm{~mm}$ absorbing coating on all baffles located at $4.2 \mathrm{~K}$ and $77 \mathrm{~K}$ to control light scattered into the camera's optics at high angles and prevent these rays from reaching the detector array. The extemal non-vacuum optics of GISMO are composed of the IRAM 30 meter primary and secondary as well as a set of flat folding and curved mirrors in the telescope's receiver cabin.

In the pre-2012 implementation of the GISMO instrument, a small folding flat mirror located $25 \mathrm{~mm}$ beneath the entrance window was used. GISMO has a cold pupil stop located in front of the $4.2 \mathrm{~K}$ silicon lens as well as a warm, sky temperature, field stop which is the IRAM secondary. With the $101 \mathrm{~mm}$ silicon lens being located $\sim 115 \mathrm{~mm}$ behind the entrance window and the input beam being $\sim \mathrm{f} / 4$, GISMO could not efficiently operate as the pre-2012 version without the use of a cold neutral density filter placed in the beam. When the opportunity arose for GISMO to have a permanent position in 2012, the external cryostat optics were modified to allow space for a cryogenic baffle extension as well as rid the design of the geometric and optical constrains imposed by the final folding mirror. This paper will detail the steps employed in the design, construction, and performance validation of the system with the cryogenic baffle extension.

Author information: Elmer Sharp; Ph: +1-301-286-4229; e-mail: clmer.h.sharp@inasa.gov 


\section{GISMO Optics before the addition of the Cryogenic Extension}

In 2011 and previous years, GISMO was set up and run from a vibration isolation table originally installed for the MaxPlanck Millimetre Bolometer (MAMBO-2) instrument. This optical configuration necessitated the use of a beam folding mirror positioned $25 \mathrm{~mm}$ below the entrance window of the cryostat as seen in Figure 1 below. The resulting cryostat configuration provided an extremely compact optical interface, however, in practice, limited the distance between the detector array, filters, and the vacuum window. This led to several design challenges associated with light scattering into the beam from high angles relative to the main beam. The resulting out of band scattered light increased the detector loading which compromised the system's mapping speed and led to low level stray optical response which ultimately limited the achievable signal to noise.

When the GISMO detector arrays were initially characterized, tests were made while the detector viewed a blackened cryogenic target in a multimode cavity. These baseline tests allowed measurement of the intrinsic detector noise and revealed that system performance was less than optimal when operated on the telescope. On the telescope, it was not possible to operate GISMO without the $40 \%$ transmission neutral density filter placed in the beam. While the window was blanked off and the detector looked into a reflective plate, the difference in power from optical loading was minimal when the cases of neutral density filter in the beam and out were compared. It was noted while lowering the plate gradually below the surface of the window and holding the plate parallel to the beam, the detector loading increased. Characterization of the camera response as a function of angle revealed that a small fraction of the light was scattered to relatively large angles that were not reflected out to the sky. The observed detector optical loading and variance indicated that this radiation was either in or close to the design band, however, a relatively small but important diffuse response to large angles was present at higher frequencies.

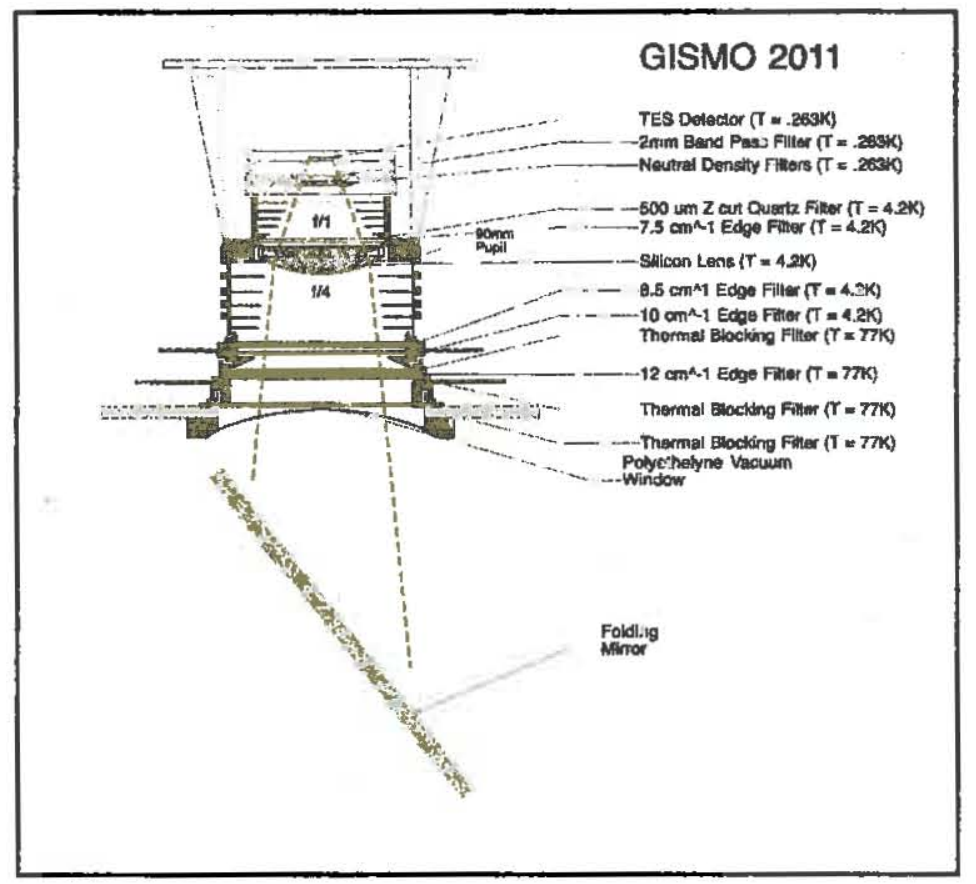

Figure 1: A 2-D drawing of the GISMO optical setup used in 2011. The detector sits in a $263 \mathrm{mK}$ environment surrounded by $2 \mathrm{~mm}$ blackened surfaces. A $40 \%$ neutral density filter is placed in the beam at the detector temperature stage. All surfaces and solid angles as seen by the detector are coated with absorptive coatings to terminate any stray light. On the bottom, looking out, side of the lens, all surfaces are coated with high emissivity materials and terminate the high angle radiation by absorbing it at either $4.2 \mathrm{~K}$ or $77 \mathrm{~K}$. A number of cold thermal blocking filters as well as band defining edge filters are placed in the beam at the different temperature stages to control the beam. 
This radiometric response was not anticipated from the initial spectral characterization of the filters elements at normal incidence. The fitters in use show high in-band efficiency in a collimated beam ${ }^{5}$. Subscquent measurements of the edge filter's spectral response as a function of angle confirmed and refined the detailed understanding of this general picture. This angularly diffuse out of band response can be understood by considering scattering from the multi-layer filters when the wavelength is less than twice the scale of the metalized patterns used in the structures. Such an effect would not be anticipated in a filter realized from a stack of homogenous layers ${ }^{8}$.

These observations pointed to the need to extend the blackened cryogenic solid angle viewed by the detector and thus enable controlled radiometric termination of this residual response. Other optical models initially could have led one to this point early on, however, the available resources, installation on the existing vibration isolation table, and optical interface set constraints on what design solutions could be easily accommodated. To carry out this plan required relocation of the instrument and a minor redesign of the optical interface. Other examples and the solutions adopted to address these concerns in the far-infrare ${ }^{9,10}$ may be of potential interest in this context.

\section{Molding and Painting Baffles}

We have developed a $2 \mathrm{~mm}$ black coating at the Goddard Space Flight center which have been used for cold calibration termination and targets, as well as cryogenic light traps similar to that used on GISMO. The millimeter wave absorptive coating is mixed from Stycast $1266^{11}$, fused Silica powder and a 25 um mesh powder of stainless steel ${ }^{12}$. Samples to test both the absorptive properties as well as the thermal contraction of the material have been fabricated and evaluated. Rods $125 \mathrm{~mm}$ in length, with varying filling fractions of fused silica were cast and immersed into liquid nitrogen to measure the thermal contraction relative to an $6061 \mathrm{~T} 6$ aluminum rod. Given that relatively thick coatings were required to provide adequate absorption, achieving a good thermal match is extremely important in ensure the material does not contract faster or slower than its mating surface. If the surfaces are not thermally matched, delimitation will occur and the absorptive coating can potentially flake off and generate debris. The coating employed was thermally matched to aluminum by appropriately tuning the volume of silica. ${ }^{13,14}$ Electromagnetically, a coating thickness of a penetration depth at the longest wavelength of interest is desirable. We have also cast the material into waveguide shim samples so they could be characterized with a Vector Network Analyzer. ${ }^{15}$ We optimized the materials physical properties to have high absorptance with minimal mass. Varying the portion of stainless steel in this mixture will determine the amount of loss in the dielectric mixture. As such, the coating can be tuned to the optimal absorption properties depending on the frequency. For GISMO, these consideration lead to the use of a mixture with $53 \%$ stainless steel by weight and cast to a thickness of $1 \mathrm{~mm}$.

The baffle design relies on multiple geometric reflections of light outside the camera's angular acceptance to control stray light. These considerations set the minimum baffle blade spacing and length. Given the coating's modest millimeter absorptance by design, one would prefer a specular finish to minimize the introduction of addition diffuse scattering from the surface. We conservatively allow for six or more bounces in the geometric optics limit. In order to minimize the number of molds used for fabrication, we fix the outer blade diameter and coat two common sets of elements, in which the inner diameter is cut into after casting. As a result of this manufacturing approach, the baffle blade spacing to length ratio is larger than required as one progresses toward the detector. From this perspective, further optimization of the baffle geometry and mass is arguably possible, however, a minimal optical risk and effort development path was pursued.

Once an appropriate mixture was identified, coating of the baffle blades with epoxy by casting was carried out. The coating has properties close to that of a fine sand mortar rather than a typical "paint" and so it must be troweled on and cast into place. For the all of the GISMO baffle blades, a machined polyethylene mold was used to control the material thickness and surface finish during casting. The Teflon, polypropylene and silicone molding compounds were initially tried, however, it was determined that all of these had limiting mechanical flaws. The Teflon was far too soft and as such the mold would disintegrate after multiple castings of the same part. The polypropylene worked well in casting but was difficult to remove from the final component and lead to breakage. The silicone casting compounds work well also but tended to disintegrate after multiple castings. As seen in Figure 2, an aluminum blank is sandwiched between two polyethylene molds and spun to high speed. The steel loaded epoxy "paint" is troweled into the center trough and spun 
up to speed repeatedly until the coating surface is evenly covered radially. Once this is done, the part is allowed to cure overnight at room temperature and released the next day.

After separating the molds from the cast part, the molds are cleaned and prepared for the next casting. In order to maintain the metal-to-metal contact region on the outside edges, some cleaning is needed on the metal surfaces since small amounts of the coating can spread to this area in the spinning process. This detail is important to enable efficient cooling of the structures upon assembly and subsequent cryogenic use. Some additional material is also applied to already cast parts where voids may have occurred in the molding process in order to maintain the $\sim 1 \mathrm{~mm}$ thickness across the baffle blade. In the case of GISMO, we had two different size blanks and two corresponding sets of polyethylene molds allowing us to cast two baffles per day. There are a total of 24 baffle blades leading to roughly 2 weeks of casting.

After all of the baffles were cast and cleaned, a CNC machine was used to cut each part to the correct beam shape depending on is position in the baffle stack. Once all of the blanks have been cut, a thin layer of the absorptive coating is applied by hand to the exposed metal edge to prevent any reflections from the exposed aluminum surface. A relatively thin, $\sim 100$ micron, layer of Aeroglaze Z306 ${ }^{16,17,18}$ is applied to all of the cast surfaces. This layer of lossy dielectric material is used to absorb mid-to-near infrared light (if present) and has the added benefit of acting as an anit-reflection coating for the cast stainless steel loaded layer. This effectively increases the coatings absorption and extends the usable range to far-infrared wavelengths required in this application. For more information on the conductively loaded epoxies formulations see discussion in Wollack et al. (2008).
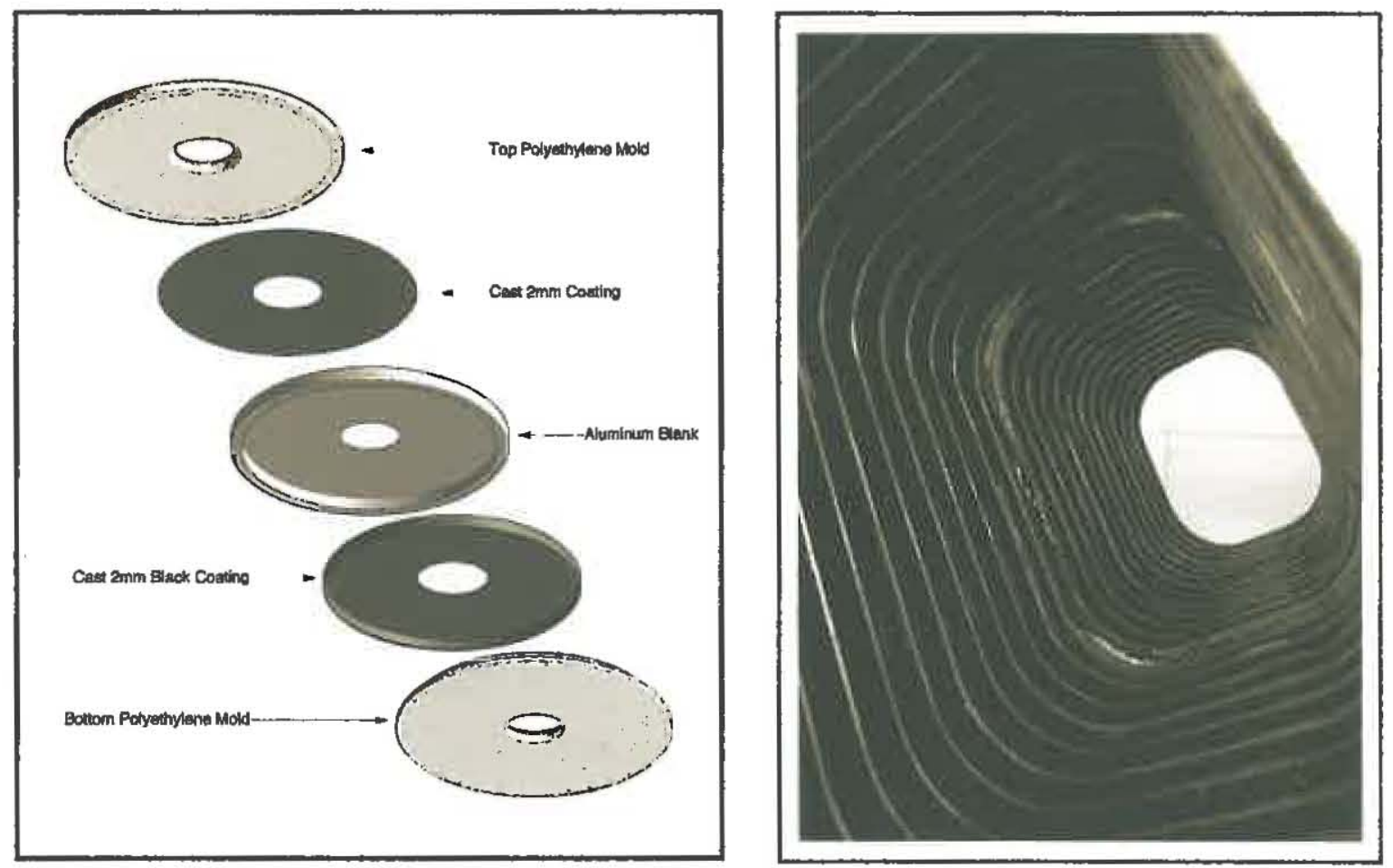

Figure 2: The cartoon on the left is representation of the casting technique used to make the GISMO baffles. An aluminum blank is placed between two polyethylene molds and spun to high speed. As the mold is spun, the stainless steel loaded epoxy is troweled into a center trough until all of the material has spread across the mold evenly. The molds are machined to leave a $13 \mathrm{~mm}$ metal edge for bolting the baffles to each other and to obtain a good metal-to-metal thermal contact area. The photo on the right is the assembled baffle stack. The holes in the baffle blades make a gradual progression from a circular (near the pupil stop and not shown in photo) to rectangular pattern which follows the evolution of the camera's field of view. 


\section{The Cryogenic Extension}

A 20 liter toroidal shaped liquid nitrogen tank built by Precision Cryogenics provides cooling to the baffle blades. The extension is $\sim 450 \mathrm{~mm} \times 380 \mathrm{~mm}$ and shares the same vacuum with the original cryostat. The tank was designed to have a step half way through the center for bolting the baffles into place and heat sinking. The 24 baffles are bolted together using 36 all-thread rods on each end. After all of the baffles have been inserted and bolted down, three temperature sensors are installed on the baffle ends and tank. The entire fixture is wrapped in the 15 layers of super-insulation to increase the hold time, blocking any extra heat leaks to this volume in order to increase the hold time.

We run the extension without any low pass filter near the window and with one thermal blocking filter located at the exit blade of the baffle stack. Thus, we try to absorb all of the out of band and high angle radiative power and terminate it rather than reflect the radiation back out the window through filtering which would have again resulted in an observed increase of stray light. (note: with a slight change to the blade and mold complexity, the thermal load could be reduced and achieve the same end effect). See Figure 3 for the final camera configuration used for the 2012 observing campaigne. We tested the cryostat with a metal reflecting plate on the entrance window and saw the bottom of the baffle stack cool to $79 \mathrm{~K}$. Upon opening the window to look into $300 \mathrm{~K}$, the bottom of the baffles began to rise in temperature and eventually settled at $85 \mathrm{~K}$. The upper thermometer in the baffle stack as well as the tank thermometer both indicated no ciange between window open and closed and remained at $77 \mathrm{~K}$.

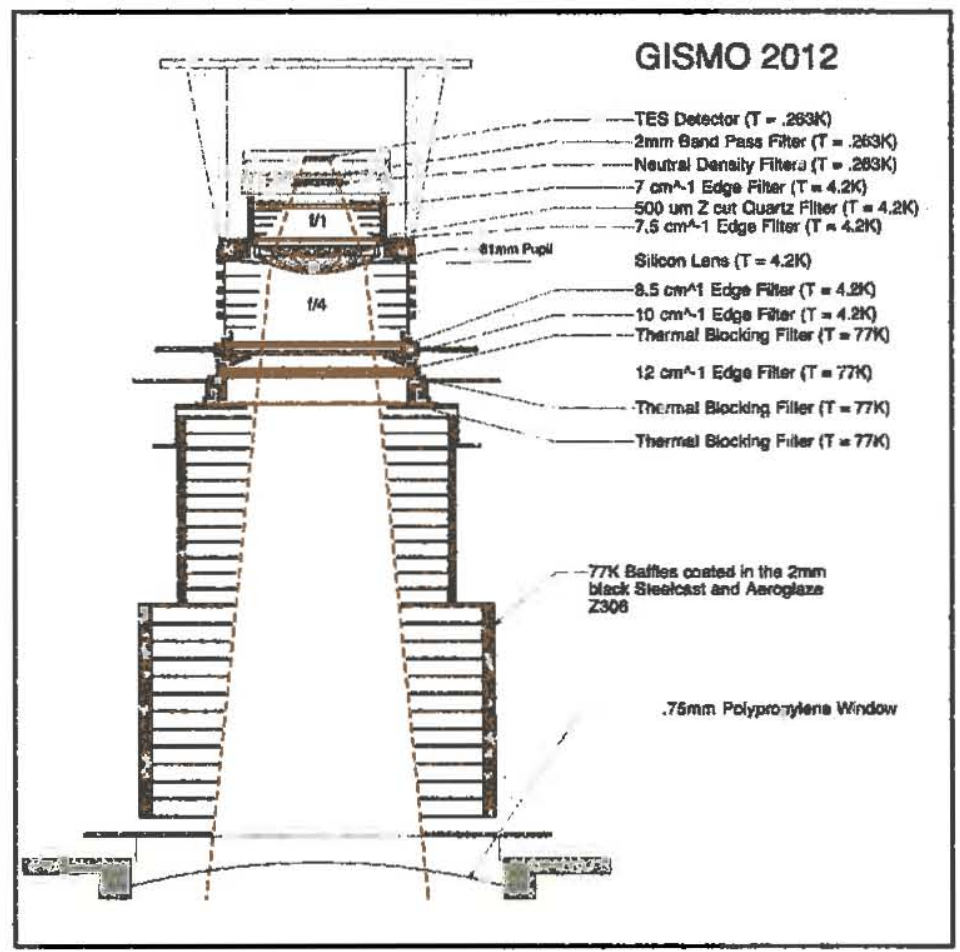

Figure 3: Shown above is a 2-D drawing of the 2012 GISMO optics internal to the cryostat. The view above is that of the short 8-pixel side of the detector, hence the smaller beam size as viewed from this angle. All of the baffles blades are coated in the $2 \mathrm{~mm}$ black stainiess steel loaded epoxy coating and painted with Aeroglaze Z306. The baffles and filters are all heat sunk to $77 \mathrm{~K}$ and $4.2 \mathrm{~K}$. The wincow material is $0.75 \mathrm{~mm}$ polypropylene and selected to maximize in-band radiation. 
A test was performed at the telescope after the integration phase of the extension to determine the new configuration performance. The temperature of the baffles in the extension were allowed to warm from $77 \mathrm{~K}$ to $102 \mathrm{~K}$ while the detector looked into a $77 \mathrm{~K}$ load. In doing so, it was observed that on average, the detector loading increased by $0.08 \mathrm{pW} / \mathrm{K}$. From this, it can be determined that we initially saw additional power, equivalent to a $13 \%$ in-band spill over to $300 \mathrm{~K}$ in the original 2011 configuration. This is equivalent to a $28.7 \mathrm{~K}$ sky temperature $(17.8 \mathrm{pW})$ recuction of power as seen by the detestor and allowed us to remove the $40 \%$ neutral density filter during 2012 observations.

The in-band beam has been restricted with the addition of the cryogenic extension and in this modified configuration, the warm spill to the receiver cabin at $300 \mathrm{~K}$ in the previous design has been terminated on the $77 \mathrm{~K}$ baffle extension. More importantly, the high frequency out of band diffuse component, which contributes a larger fraction to the observed excess detector loading and noise, has been mitigated. See Figure 5 for a simple geometric ray trace for the input filter stack. In the 2011 observing season, GISMO's Noise Equivalent Flux Density (NEFD) was $\sim 16 \mathrm{~mJ} / \sqrt{\mathrm{Hz}}$. After the extension was added, the NEFD was reduced to $\sim 10 \mathrm{~mJ} / \sqrt{\mathrm{Hz}}$ and was limited by variations in atmospheric brightness.

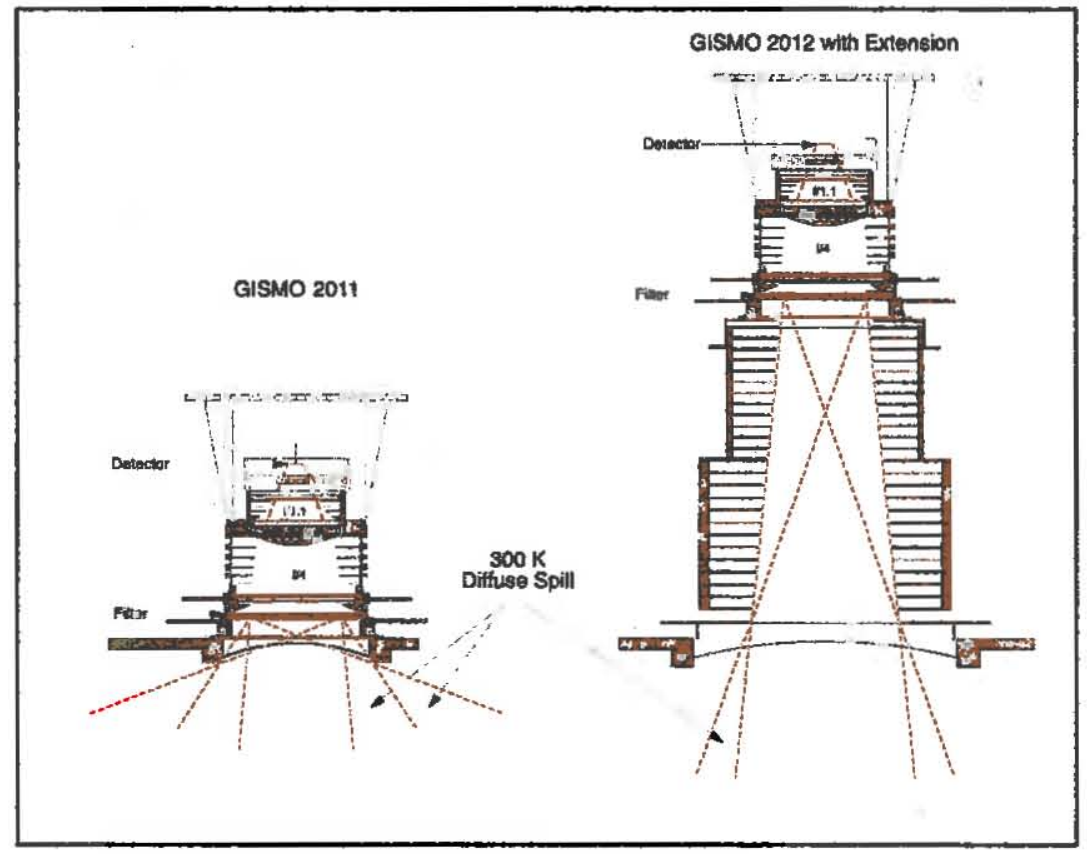

Figure 5: A side-by-side comparison of the input-solid-angle to the first edge filter in the GISMO filter stack. The extent of filter stack's input-solid-angle is indicated by the dashed lines. By increasing the baffle length, the field of view of the detector and the filters approach $\sim 30$ degrees and eliminates the excess power loading observed in the initial design due to large angle diffuse scattering to $300 \mathrm{~K}$. The baffle aperture transforms from circular to rectangular and the image corresponds to the 8-pixel (shorter) side of the array. The window diameter is set by the need for a path free of vignetting for the array's diagonal response.

\section{Conclusions}

Stray light can enter the optical path from unintended scattering paths, ghosting from anti-reflection coatings, diffractive spill, and many other sources. In this case, a small out of the band scattering component to large angles provided a path for cut of band thermal emission to load the detector. Initially, this restricted GISMO to use a $40 \%$ transmission neutral density filter to prevent detector saturation during observations. A solution to reduce this component of the stray light observed by the GISMO receiver has been demonstrated. A $2 \mathrm{~mm}$ black coating was highly effective with $\sim 85 \%$ absorption per bounce at $2 \mathrm{~mm}$ was used in the construction of a cooled baffle assembly. The overall NEFD was lowered and thus increased our mapping speed by nearly a factor of 3 . 


\section{Acknowlegments}

The authors would like to thank P. W. Cursey for his guidance and support in machining.

\section{References}

[1] J.G. Staguhn, D. Benford, R.G. Arendt, D.J. Fixsen, A. Karim, A Kovacs, S. Leclercq, S.F. Maher, T.M. Miller, S.M. Moseley, E.J. Wollack, "Latest Results from GISMO: a 2-mm Bolometer Camera for the IRAM 30-m Telescope", 2011, EAS Publications Series, M. Röllig, R. Simon, V. Ossenkopf and J. Stutzki (eds), Conditions and Impact of Star Formation, Vol. 52, pp. 267-271.

[2] E.H. Sharp, D.J. Benford, D.J. Fixsen, S.F. Maher, C.T. Marx, J.G. Staguhn, E.J. Wollack, "Design and performance of a high-throughput cryogenic detector system," 2008, Millimeter and Submillimeter Detectors and Instrumentation for Astronomy IV, Proceedings of the Society of Photo-optical Instrumentation Engineers (SPIE), Vol. 7020, p. L202.

[3] C.A. Allen, D.J. Benford, J.A. Chervenak, D.T. Chuss, T.M. Miller, S.H. Moseley, J.G. Staguhn, E.J. Wollack, "Backshort-Under-Grid arrays for infrared astronomy" 2006, Nuclear Instruments and Methods in Physics Research A, $559,522$.

[4] J. Lau, J. Fowler, T. Marriage, L. Page, J. Leong, E. Wishnow, R. Henry, E. Wollack, M. Halpern, D. Marsden, G. Marsden, "Millimeter-Wave Antireflection Coating for Cryogenic Silicon Lenses," 2006, Applied Optics, Vol. 46, pp. 3746-3751.

[5] P. Ade, G. Pisano, C. Tucker, and S. Weaver, "A review of metal mesh filters," Proc. SPIE 6275, 62750U (2006).

[6] C. E. Tucker and P. A. R. Ade, "Thermal filtering for large aperture cryogenic detector arrays," Proc. SPIE 6275, 62750T (2006).

[7] P. Goldsmith, "Quasi-Optical Systems: Gaussian Beam Quasi-Optical Propagation and Applications" 1998, IEEE Press, New York.

[8] H.A. MacLeod, "Thin-Film Optical Filters," $3^{\text {rd }}$ Ed., 2001, Institute of Physics Publishing, London, pp. 283-292.

[9] C.D. Dowell, C.A. Allen, S. Babu, M.M. Freund, M.B. Gardner, J. Groseth, M. Jhabvala, A. Kovacs, D.C. Lis, S.H. Moseley, T.G. Phillips, R. Silverberg, G. Voellmer, H. Yoshida, "SHARC II: A Caltech submillimeter observatory facility camera with 384 pixels", 2003, Millimeter and Submillimeter Detectors for Astronomy. Edited by Phillips 4855, 73-87; Dowell, C.D. (JPL), private communication, March 16, 2011, (note: after commissioning as described in this reference, the filter stack position was relocated to lower end of baffle assembly to reduce input solid angle to the filter. This migrated the observed detector power loading encountered during telescope observations.).

[10] R. Dunner, P. Gallardo, E.J. Wollack, F.J. Henriquez, C. Jerez, "Mirror illumination and spillover measurements of the Atacama Cosmology Telescope", 2012, SPIE Astronomical Telescopes and Instrumentation, Amsterdam, Netk:erlands; (see these proceedings).

[11] Stycast 1266, Emerson \& Cuming, 46 Manning Road, Billerica, MA 01821, USA.

[12] Micro-Melt, 25 micron mesh stainless steel powder; Carpenter Powder Products Inc., 600 Mayer Street, Bridgeville, PA 75017.

[13] N. Marcuvitz, "Waveguide Handbook", 1951, MIT Rad. Labs. Series, Vol. 10, McGraw Hill, New York.

[14] E.J. Wollack, D.J. Fixsen, A. Kogut, M. Limon, P. Mirel, J. Singal, "Radiometric Waveguide Calibrators," 2007, IEEE Transactions on Instrumentation and Measurement, Vol. 56, No. 5, pp. 2073-2078.

[15] E.J. Wollack, D.J. Fixsen, R. Henry, A. Kogut, M. Limon, P. Mirel, "Electromagnetic and Thermal Properties of a Conductively Loaded Epoxy", 2008, International Journal of Infrared and Millimeter Waves, Vol. 29, No. 1, pp. 51-61.

[16] Aeroglaze Z306, Lord Corporation, 111 Lord Drive, Cary, NC 27511, USA. 
[17] M.J. Persky, "Review of Black Surfaces for Space-Borne Infrared Systems", 1999, RSI, Vol. 70, No. 5, pp. 21932217.

[18] L. Kauder, "Spacecraft Thermal Control Coatings References", 2005, NASA GSFC, Technical Report, NASA/TP2005-212792. 